\title{
Examination of advanced fastening systems for the use of robots in the construction industry
}

\author{
Panagiotis Spyridis $^{\mathrm{a}, *}$, Arnim J. Spengler ${ }^{\mathrm{b}}$, Dirk Schlüter ${ }^{\mathrm{b}}$, Tobias Bruckmann ${ }^{\mathrm{c}}$, \\ Alexander Malkwitz \\ ${ }^{a}$ Chair of Fastening Technology, Department of Architecture and Civil Engineering, TU Dortmund University, August-Schmidt-Straße 8, \\ Dortmund 44227, Germany \\ ${ }^{b}$ Institute of Construction Management, Department of Civil Engineering, Faculty of Engineering, University of Duisburg-Essen, \\ Universitätsstraße 15, 45141 Essen, Germany \\ ${ }^{c}$ Chair of Mechatronics, Department of M echanical and Process Engineering, Faculty of Engineering, University of Duisburg-Essen, \\ Universitätsstraße 15, 45141 Essen, Germany
}

\begin{abstract}
Since robotics become more widespread in the construction industry, more construction phases and work steps must be covered with one robot system. Current robot systems are used almost exclusively in precast construction. At the construction site, so far only prototypes are in use, and only individual parts of the building shell construction and assembly can be covered. This paper examines to what extent fastenings are necessary to increase the operating range of robots and which boundary conditions exist or need to be addressed. Automated construction, and more precisely installation of fastenings, has so far been partially implemented, which has shown increased productivity as well as installation quality, thus load-bearing safety. This knowledge must now be extended to robots. The present work is based on an overview of current research and development and on the current research on a cable robot for brickwork construction at the university Duisburg-Essen. It further demonstrates that fastenings pose an important further application, especially in order to explore the extended phases towards the brickwork or contour crafting. It can be assumed that robots will become increasingly important in the construction industry. Reasons include high quality, safety, speed and economic aspects.
\end{abstract}

(C) 2019 The Authors. Published by Budapest University of Technology and Economics \& Diamond Congress Ltd.

Peer-review under responsibility of the scientific committee of the Creative Construction Conference 2019.

Keywords: Advanced fastening systems; Fixings and fastenings; Robots in the construction industry; Automatisation/Automation

\section{Introduction}

Robot systems in the construction industry have been researched since the early 1980s. Since then, the use of robots has first been established in factories for precast structural elements. These systems are used here, as in other areas of stationary production as well, for many consistent tasks. Contrary to this, robot systems have not been able to assert on the construction site to this day as discussed in previous publications [1], [2], [3]. A possible reason for this is the reliance of the construction industry on $2 \mathrm{D}$ model prints, as well as unique project/product specifications, which limits

*Corresponding author: Author email: panagiotis.spyridis@tu-dortmund.de 
Spyridis et al. / Proceedings of the Creative Construction Conference (2019) 046

https://doi.org/10.3311/CCC2019-046

the mobility programming of robots. Another reason is the limited working envelope of machines, i.e. lack of free movement systems for a robot along a wide part of the construction site.

However, these constraints seem to experience a period of transformation in the last years, while assembly of construction elements may be largely facilitated [4], [5]. This paper describes the requirements and possible methods in the construction industry as regards the installation of fixings and assembly of fitted elements by robots. On this basis, the further research and applicability potential and necessity are inquired, and directions for further investigations are established. In the following, the importance of fixations in the described area of application is introduced. Then, some construction tasks and objectives with regards to fastenings are presented, with emphasis on those which a robot system can perform better than humans under specific conditions. Also, other reasons for considering the implementation of robots for these tasks are discussed, such as e.g. health and safety at work, quality, or avoidance of defects due to human errors.

\section{Presentation of robot systems in the building industry}

Before going on to fixations, a brief outline of robotics in the construction industry is given. This is necessary to understand the open questions.

In the 80's of the previous century for the first time the topic of the automated construction of buildings was discussed in a larger circle. At that time, the portal robot has been considered to be particularly suitable for the construction of buildings. Until today, these types of robots are often used, because they can bridge high spans. Today many of the earlier 2000 robot systems can be found in precast factories. Robot systems, which can be installed directly on the construction site, were not followed up during the conception phase. Some concepts considered an automated approach where the fixations are inserted from above and the covered with concrete. However, many do not address this topic at all, or these activities are finally still manually carried out. Examples are the construction robots in Japan, where the reinforcement is interlaced manually. These activities will also continue to be performed manually for the systems presented in Table 1.

Table 1: Systems, basic platforms and manufacturing processes for (partially) autonomous building construction

\begin{tabular}{lll}
\hline Name & Basis platform & manufacturing method \\
\hline Apis Cor & Modified robot arm & Contour Crafting \\
\hline Hadrian & Modified crawler excavator & brickwork \\
\hline Hadrian X & Modified truck with cantilever arm & brickwork \\
\hline SAM & Robot arm & brickwork \\
\hline Wirerobot for contruction & Wire robot & brickwork \\
\hline Spiderbot & Wire robot & Contour Crafting \\
\hline Kamermaker & Portal robot & Contour Crafting \\
\hline Vulcan + Vulcan II & Portal robot & Contour Crafting \\
\hline
\end{tabular}

From a construction point of view, the manufacturing processes are particularly interesting. The above shown systems can only be used to a limited extend of the building shell construction. Thus, the actual systems are limited to parts of the building shell and cannot be used to other construction methods. As a result, many of the extended construction activities like the insertion of windows or setting the roof truss cannot currently be automated using these systems.

When robot systems are to be used in the extended bodyshell and set fixings, new challenges arise:

- It is not possible to use the same mechanism for setting fastenings like e.g. building with concrete. 
Spyridis et al. / Proceedings of the Creative Construction Conference (2019) 046

https://doi.org/10.3311/CCC2019-046

- The usage of several robot types is a cost driver, which currently leads to a point of continuing with an existing robot system, while modular approaches are not applicable.

- It must be decided whether first the fixings and then the component should be set or both at the same time.

- Clarification is needed as to if conventional or not conventional standardized fixing products can be used

- New fixing methods for usage in robot driven systems need to be standardised

- The construction process must remain continuous and undisturbed

\section{Parameters that determine the use of fixtures by robot systems in the construction industry}

In addition to the challenges just described, there are further parameters affecting the usage of robot systems. The following list gives an overview:

Heaviness: The high weight of robot systems makes it difficult to use them on site. For installation, the subfloor must be sufficiently load-bearing, installation on floor slabs is made more difficult or is not possible, and adequate stability must be demonstrated.

Speed: The speed of the overall system for moving components has a direct influence on the overall construction time. This includes the procedure or moving of the base platform, as well as the speed of the end effector.

Complexity: The complexity is conditioned by properties such as: Operation, construction, programming and the preprocessing.

Payload: A low payload means the displacement of smaller components and a shorter construction time. Some components require a minimum payload.

Radius of action: A small radius of action requires frequent repositioning of the robot system. Moving the entire system is at the expense of the construction speed.

Height limitation: The systems should be able to erect several storeys without having to dismantle and erect them at great expense.

Sensitivity to environmental influences: In order to be used on the construction site, sufficient insensitivity to environmental influences should be ensured. The systems should be insensitive to dust, heat, cold and water. Mechanical shocks are to be expected.

Costs of use: The operational costs of the overall system have a direct influence on the economic efficiency and the operational hurdle for companies.

In addition, simulations have shown that the usage of robots only in shell construction is not economically efficient. The systems must be used in further construction phases. In these phases the setting of fixtures becomes of essential importance. Work in the extended shell construction phase can include various fittings, such as the installation of facade elements, balconies, windows, door frames, HVAC parts, or receptors for building expansion. For these installations, advanced fastening systems potentially with adaptable or generic assembly features are important, and robots can be used more efficiently, the more work breakdown structure (WBS) elements they can be involved with. In the following, current conventional fixing types are described.

A large variety of construction products (post-installed or cast-in-place fasteners) together with adaptably designed fixtures for various building configurations and uses represent modern fastening technology. Furthermore, a consistent set of product performance documents [6] and structural design calculation standards [7] form the basis for specifying, detailing, and installing fastenings. The large variety of products in the market (see an exemplary presentation in Fig. 1) aims to satisfy a variety of performance requirements with considerations on minimising costs and assembly speeds. These requirements include available dimensional configurations, load-bearing capacities, and wider project 
Spyridis et al. / Proceedings of the Creative Construction Conference (2019) 046

https://doi.org/10.3311/CCC2019-046

specifications such as thermal and noise insulation, fire resistance, durability, environmental footprint, aesthetics, and ease of removal. Post-installed anchors are mostly available for use in concrete and masonry, while some applications of post-installed anchors for steel structures, wood, and other construction materials are also available in the market. Although each anchor has individual installation procedures, the generic steps for anchor installation can be described as follows:

- Marking of fastening point

- Drilling of borehole (perpendicularly and using the appropriate drilling tools)

- Cleaning of borehole (removal of drill dust)

- Preparation/mixing of injection mortar (for bonded anchors)

- Insertion of anchor at appropriate depth

- Tightening of nut to the correct torque

- Optionally: load-testing of anchor

Furthermore, mostly depending on the nature of the fixture, and the construction plan, two different types of fixture assembly can be used as seen in Fig. 2. The assembly with pre-positioned anchors requires that all the above steps up to anchor installation occur prior to placing and securing the fixture. This requires that the drilled hole in the base material is larger than the clearance hole in the fastened element. Simultaneously, this requires a very precise positioning of the anchor in order to avoid clashes with the fixture holes. Post installation configurations use the element to be fastened as a drilling and setting template for the anchors. In this case, the diameter of the hole in the component to be fastened should be larger than or equal to the borehole diameter. In this case, it is also important to provide for a safe temporary support of the fixture during the anchor setting procedure. A practical method - though not always applicable - is mixing the above methods by temporarily supporting the fixture with one pre-positioned anchor and subsequently installing the rest of the anchors. An issue with post-installation is the possible damage of the fixture due to the through-drilling procedures, which compromises the appearance and the durability of the system. According to the authors' best knowledge, post-installation is still a preferred method for most applications in the field.

\section{Requirements for advanced fastening systems}

Prefabrication, automated erection, and modularisation procedures gain in relevance hand in hand with the increasing implementation of digital construction technologies. Besides the parameters of robotised construction discussed above, the use of automated procedures can generally minimise or eliminate typical health and safety risks related to the installation of fixings. These include hand injuries, among other due to drill torque reaction, hand arm vibrations, inhaling of dust, high noise levels, working with chemicals, electric shocks. Robotised construction can also facilitate the installation of fixings where human presence is of unacceptable high health and safety risks or virtually impossible. These cases include installations in very confined spaces, work at height, underwater installations, construction at extreme environmental or space conditions, rehabilitation or decommission of industrial and nuclear facilities.

Besides, it is widely proven that human errors in the installation of fixings can substantially decrease the load bearing capacity of the anchor, occasionally even down to $20 \%$ of the expected resistance. Simultaneously, statistics and industry reviews have shown that perhaps the majority of construction professionals are unaware of the exact installation procedures for fixings [8], [9]. A faulty installation can lead to reduced structural safety already at the beginning of the fastenings life-cycle but defects at installation can also propagate to a faster degradation of the fixing and possibly a failure without prior warning [10]. These facts obviously lead to very high technical risks, and this has already led to catastrophic collapses in the past. In order to ensure quality of construction and a permanently high safety level, robotized construction, where human quality control is replaced by automated procedures. 
Spyridis et al. / Proceedings of the Creative Construction Conference (2019) 046 https://doi.org/10.3311/CCC2019-046
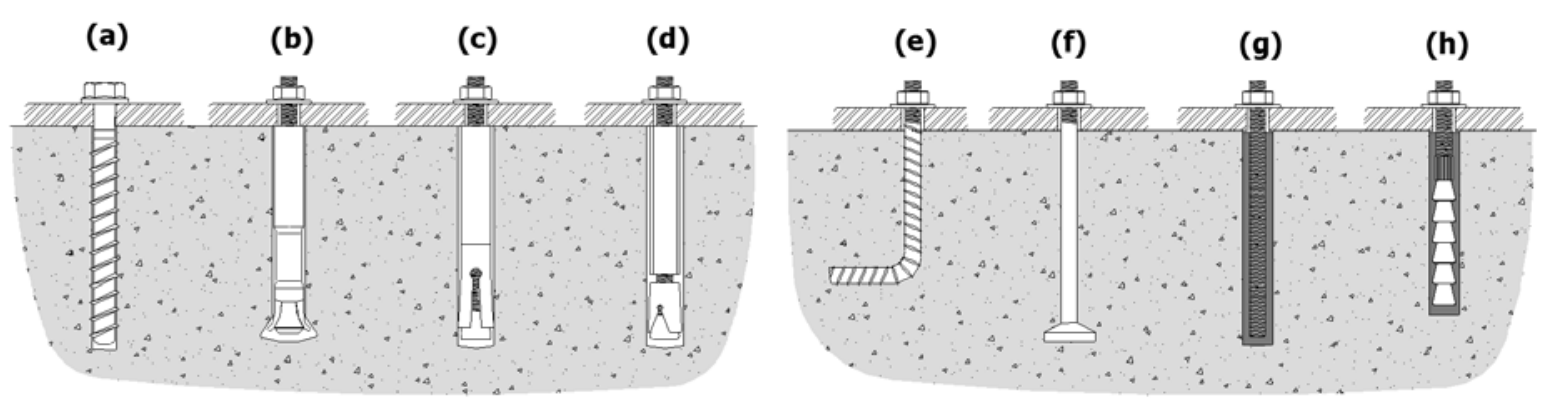

Fig.1. Different anchor types, depending on the installation procedure: (a) concrete screw, (b) undercut anchor, (c) expansion anchor - sleeve type, (d) expansion anchor - bolt type, (e) cast-in-place ribbed and deformed bar, (f) cast-in-place headed stud, (g) bonded anchor with threaded rod, (h) special bonded anchor.

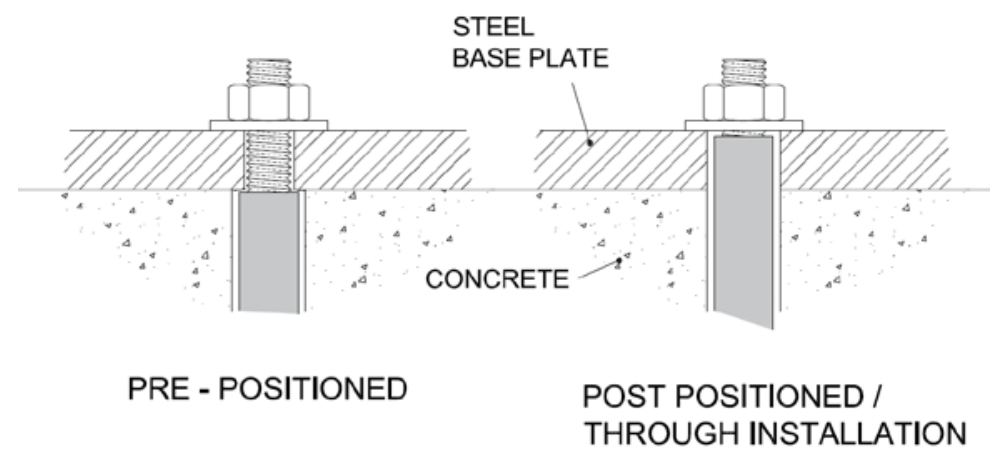

Fig. 2. Anchor installation configurations, dependent on logistic

It should also be mentioned that current design codes propose an additional design safety factor for fixings to account for the installation quality on the load bearing capacity as discussed in the previous paragraph [11]. This safety factor increases the design demand by $40 \%$ for less than normal installation safety (e.g. untrained or inexperienced personnel). This also shows a directly proportional increase as regards all aspects of fixings installation such as procurement cost, time, and material resources.

As regards positioning of the anchor, an often-recurring problem is drilling onto or through rebar in concrete. In this case, both the function of the anchor and the efficiency of the reinforcement can be compromised, and as such the borehole is cancelled and a new one must be drilled, at a minimum distance of a few centimetres. This means that the fixing point must also be redesigned. In some cases, this is also due to over- or under-drilling. In order to avoid this effect, the as built drawings, and a ferromagnetic rebar scanner are employed. Tolerances also play a very important role in fastening technology, as they can be decisive for the success of the assembly. Furthermore, tolerances can strongly influence the distribution of loads from the fixture to the individual anchors, e.g. in a group, and consequently lead to excessive load on a single anchor [12]. In absence of redundancy measures, failure of a single anchor can then even lead to collapse of the entire fixture.

The requirements for the fixing system used for an automated assembly are derived from the above descriptions. In summary, specific rules and methods need to be set as regards the interfaces and integration of fastening products with existing robot systems. Also, the generic steps for anchor installation must be reproducible, and insensitive to the location, or variabilities as regards the base material, fixture, or intended use. The fixing product should comply with existing standards, while it should be noted that these standards (typically the certified manufacturer's declaration of performance) dictate the construction/installation method. Finally, it should obviously provide the logistical benefits in order to not negatively affect the speed and construction sequence of the system. 
Spyridis et al. / Proceedings of the Creative Construction Conference (2019) 046 https://doi.org/10.3311/CCC2019-046

An ideal application would allow the robot system to immediately integrate the complete fastened system (i.e. fixing and fixed component) into the building. This requires a fixation method that accommodates this from a load-bearing and a logistic viewpoint. If the fixing installation is nonetheless performed manually, a robot system should be able to control whether they have been made correctly, e.g. by on site testing and evaluation.

\section{Conclusion}

This study addresses the use of robots in construction. It departs from extended phases of brickwork or contour crafting, toward the potential and efficiency that can be achieved when robotic installations are developed for fastenings and assemblies of fitted components in construction. This further potential of robotized construction applications is discussed on the basis of particularities and benefits of robotic construction, current obstacles and risks in manual fastening installations, and how these two aspect of construction can be integrated toward a safe and efficient practice.

Based on this exercise, the efficiency of automated fastening assemblies is discerned, while significant new research questions arise. These questions also pose the challenges for research investigations and development of efficient technologies. As regards first applications of robotic systems, the University of Duisburg-Essen is currently developing a cable robot and performs investigations for brickwork construction [13]. The involved scientific teams envisages using this robot system to address the particularities of fastening applications for specific building systems, with appropriate adjustments and extensions of the present system. In order to provide suitable systems to this end, further research is necessary, along the axes of the open questions briefly listed below:

- Which robot types and end effector types are potentially usable for the installation of fastenings and fitted components?

- In which construction phases can automated fixation solutions be used?

- Why current robot systems do not set fixations in the construction industry?

- How do other industries install automated fastenings?

- Which design and construction standards are involved and how should these be adapted?

Once these research questions are addressed, a knowledge basis will become available in order to translate the integrated automated fastening assembly into a real construction system.

\section{References}

[1] L. Cousineau, N. Miura. Construction robots: the search for new building technology in Japan. ASCE Publications, American Society of Civil Engineers, 1998.

[2] T. Bruckmann, H. Mattern, A. Spengler, C. Reichert, A. Malkwitz, M. König, Automated construction of masonry buildings using cable-driven parallel robots. In ISARC. Proceedings of the International Symposium on Automation and Robotics in Construction (Vol. 33, p. 1). Vilnius Gediminas Technical University, Department of Construction Economics \& Property, 2016.

[3] T. Bruckmann T, C. Reichert, M. Meik, P. Lemmen, A. Spengler, H. Mattern, M. König. Concept Studies of Automated Construction Using Cable-Driven Parallel Robots, Cable-Driven Parallel Robots, Springer (2018) pp. 364-375. http://dx.doi.org/10.1007/978-3-319-61431-1_31

[4] P. Vähä, T. Heikkilä, P. Kilpeläinen, M. Järviluoma, E. Gambao, Extending automation of building construction-Survey on potential sensor technologies and robotic applications. Automation in Construction, 36, (2013), pp. 168-178. https://doi.org/10.1016/j.autcon.2013.08.002

[5] B.V. Viscomi, W.D. Michalerya, L.W. Lu, Automated construction in the ATLSS integrated building systems. Automation in Construction, 3(1), (1994), pp. 35-43. https://doi.org/10.1016/0926-5805(94)90030-2

[6] Eropean Parliament and Council, EU Regulation No. 305/2011 Construction Products Regulation (CPR), Brussels, 2011.

[7] Comité Européen de Normalisation (CEN), EN 1992-4:2018. Eurocode 2: Design of concrete structures - Part 4: Design of fastenings for use in concrete. Brussels, Belgium, CEN, 2018.

[8] P. Grosser, W. Fuchs, R. Eligehausen, A field study of adhesive anchor installations. Concrete international 33(1), (2011) pp. 57-63.

[9] B. Cronyn, Industry fears over fixings, New Civil Engineer (Septemeber), 2015.

[10] Construction Industry Research And Information Association - CIRIA. General fixings Guidance on selection and whole life management C777. CIRIA, London, 2019

[11] fib - The International Federation for Structural Concrete, fib Bulletin 58: Design of anchorages in concrete. Guide to good practice, 2011

[12]P. Spyridis, K. Bergmeister, Effects of Assembly Tolerances on Bolted Anchorages in Concrete, Journal of Structural Engineering 140(1), (2012), p. 04013024 https://doi.org/10.1061/(ASCE)ST.1943-541X.0000776

[13] A. Malkwitz, A. Spengler, T. Bruckmann, Baubetriebliche Untersuchung von Robotersystemen im Mauerwerksbau (Investigation of robot systems in masonry construction). Bautechnik, 96(4), 2019. 